\title{
Analyzing folding and degradation of metabolically labelled polypeptides by conventional and diagonal sodium dodecyl sulfate-polyacrylamide gel electrophoresis
}

\author{
Tatiana Soldà ${ }^{1}$, Silvia Olivari ${ }^{1}$ and Maurizio Molinari ${ }^{1^{\star}}$
}

\begin{abstract}
1Institute for Research in Biomedicine, Università della Svizzera italiana-USI. CH-6500 Bellinzona, Switzerland.
*Corresponding Author: Maurizio Molinari, Institute for Research in Biomedicine, Università della Svizzera italiana-USI. CH-6500 Bellinzona, Switzerland. Email: maurizio.molinari@irb.unisi.ch
\end{abstract}

Submitted: June 16, 2005; Revised: July 14, 2005; Accepted: September 20, 2005.

Indexing terms: Calnxexin; Endoplasmic Reticulum; Molecular Chaperones; Protein Folding.

Abbreviations: BACE, beta secretase; CHAPS, 3-(3-cholamydopropyl)dimethylamino)-1-propanesulphonate; Cnx, calnexin; ER, endoplasmic reticulum; ERAD, ER-associated protein degradation; HA, influenza virus hemagglutinin; SDS-PAGE, sodium dodecyl sulfate-polyacrylamide gel electrophoresis.

\begin{abstract}
Efficient protein folding and quality control are essential for unperturbed cell viability. Defects in these processes may lead to production of aberrant polypeptides that are either degraded leading to "loss-of-function" phenotypes, or deposited in or outside cells leading to "gain-of-toxic-function" phenotypes. Elucidation of molecular mechanisms regulating folding and quality control of newly synthesized polypeptides is therefore of greatest interest. Here we describe protocols for metabolic labelling of transfected/infected mammalian cells with [ ${ }^{35 S}$ ]-methionine and [ $\left.{ }^{35} \mathrm{~S}\right]-$ cysteine, for immunoisolation from detergent extracts of the selected model proteins and for the investigation of the model polypeptide's intracellular fate in response to chaperone-deletions or to cell exposure to folding or degradation inhibitors.
\end{abstract}

\section{INTRODUCTION}

Secretory proteins, luminal or membrane-associated proteins of the endo-exocytic compartments and plasma membrane proteins are expressed by ribosomes associated with the cytosolic face of the proteinconducting Sec61 channels in the ER membrane. The nascent chains are injected in the ER lumen and their folding is assisted by a large number of ER-resident molecular chaperones and folding factors (1). The coand post-translational addition of oligosaccharides on asparagine residues (N-linked glycosylation (2)) and the formation of disulphide bonds covalently linking the polypeptide's cysteines (3) are crucial for acquisition of native architecture. Completion of the folding process may also involve formation of homo- or heterooligomeric complexes that normally precedes release of the native proteins from the ER and transport at their final intra- or extracellular destination $(4,5)$. Protein folding is errorprone and gene deletions or mutations may further reduce folding efficiency. Folding-defective polypeptides are retained in the ER lumen. To avoid accumulation of these toxic by-products of protein biosynthesis that could trigger unfolded protein responses often resulting in cell death (6), aberrant polypeptides are eventually dislocated back into the cytosol for proteasome-mediated degradation $(7,8)$. This article describes an approach that has been instrumental to analyze in living cells the molecular mechanisms regulating protein folding and disposal, namely, the transient associations of immature polypeptides with ER-resident chaperones (9-11) and enzymes (12), the formation of intra- and intermolecular disulfide bonds as essential steps in the protein folding process (13-15), the folding or degradation kinetics in the 
presence of cell permeable inhibitors or in cells depleted of selected ER-resident chaperones $(16,17)$. The approach consists of six distinct steps: 1) amplification of the signal by highest synthesis of the protein-of-interest (cell transfection or infection); 2) metabolic labelling of nascent polypeptides expressed in living cells followed by incubation in non-radioactive medium to allow maturation/degradation of the protein-of-interest to proceed (pulse-chase); 3) "freezing" of the labelled polypeptides in a state reflecting their situation at given times after synthesis (sample alkylation and cell lysis); 4) isolation of the protein-of-interest or of complexes containing protein-of-interest:ER-resident chaperones with specific antibodies (immunoprecipitation); 5) Electrophoretic analysis of the samples by conventional SDS-PAGE or by 6) diagonal SDS-PAGE.

\section{MATERIALS AND METHODS}

Cell media: DMEM (Gibco), 10\% fetal calf serum (FCS), $1 \%$ penicillin and streptomycin (PS)

PBS: $20 \mathrm{mM}$ sodium dihydrogen orthophosphate, 100 $\mathrm{mM}$ sodium chloride, $80 \mathrm{mM}$ di-sodium hydrogen orthophosphate anhydrous, $\mathrm{pH} 7.4$

Transfection media: DMEM (Gibco), 10\% FCS, 1\% nonessential amino acids (Gibco), Optimem (Gibco)

Transfection reagent: Lipofectamine ${ }^{\mathrm{TM}} 2000$ (Invitrogen)

Inoculum: Serum-free RPMI (Gibco), 20 mM HEPES $\mathrm{pH}$ $6.8,0.2 \%$ bovine serum albumin (BSA), $10-30$ plaqueforming units (pfu) of virus/cell

MG132 (Inhibits ERAD by inactivation of the cytosolic proteasome): Stock $10 \mathrm{mM}$ in DMSO. To be used at a final concentration of $50 \mu \mathrm{M}$

Kifunensine (Inhibits ERAD by inactivation of the ER alpha-mannosidase I): Stock $20 \mathrm{mM}$ in $\mathrm{H}_{2} \mathrm{O}$. To be used at a final concentration of $100 \mu \mathrm{M}$

Starvation medium: DMEM w/o L-methionine, w/o Lcystine, $50 \mathrm{mM}$ HEPES $\mathrm{pH} 7.4$
Pulse medium: Starvation medium supplemented with $0.1 \mathrm{mCi} / \mathrm{ml}\left[{ }^{35} \mathrm{~S}\right]-$ methionine and $\left.{ }^{35} \mathrm{~S}\right]$-cysteine (Redivue 35S-Promix, Amersham)

Chase medium: DMEM, $50 \mathrm{mM}$ HEPES, $5 \mathrm{mM}$ cysteine, $5 \mathrm{mM}$ methionine

Stop solution (protein alkylation): $20 \mathrm{mM} \quad \mathrm{N}-$ ethylmaleimide (NEM) in PBS

Protease inhibitors cocktail (CLAP): $5 \mathrm{mg}$ chymostatin, $5 \mathrm{mg}$ leupeptin, $5 \mathrm{mg}$ antipain, $5 \mathrm{mg}$ pepstatin A in 500 $\mu \mathrm{l}$ DMSO (stock 1000X)

Phenylmethylsulfonyl fluoride (PMSF): Stock $200 \mathrm{mM}$ in isopropanol. To be used at the final concentration of 1 $\mathrm{mM}$

HBS: 50 mM HEPES, $200 \mathrm{mM} \mathrm{NaCl}$ (pH 6.8 and pH 7.4)

Lysis: 2\% CHAPS in HBS pH 6.8, 1x CLAP, 20 mM NEM, $1 \mathrm{mM}$ PMSF

ProteinA-Sepharose beads (PAB): 10\% w/v suspension of PAB (Sigma-Aldrich) in HBS pH 7.4

Washing Buffer: 0.5\% CHAPS in HBS pH 7.4

Sample buffer: $82 \mathrm{mM}$ TRIS, $10.5 \%$ Glycerol, $2 \%$ SDS, $0.001 \%$ Bromophenol blue, $\mathrm{pH}$ 6.8. Sample buffer is supplemented with $100 \mu \mathrm{M}$ fin DTT for reducing gels

Coomassie blue stain: $0.25 \%$ Coomassie blue, $50 \%$ methanol, $7.5 \%$ acetic acid

Destain: 20\% methanol, $7.5 \%$ acetic acid

ImageQuant software: Amersham Biosciences

Film: Eastman Kodak BioMax MR

\section{RESULTS AND DISCUSSION}

This article describes a pulse-chase approach used in combination with immunoprecipitation and conventional or diagonal SDS-PAGE to investigate protein folding in the ER and disposal of folding-

Soldà et al. - Analyzing folding and degradation of metabolically labelled polypeptides by conventional and diagonal sodium dodecyl sulfate polyacrylamide gel electrophoresis

www.biologicalprocedures.com 
defective polypeptides from the ER of mammalian tissue culture cells.

\section{Folding of influenza HA in mouse embryonic fibroblast}

HA is a type I transmembrane glycoprotein with multiple folding domains. In infected cells the ectodomain is cotranslationally translocated into the ER where N-linked glycosylation of nascent chains does occur. HA oxidative folding starts co-translationally and terminates post-translationally with oxidation of the last Cys14-Cys466 disulphide. HA oxidation can be monitored by taking advantage of differences in electrophoretic mobility in nonreducing SDS-PAGE between the native HA form (NT) and forms with an incomplete set of disulfide bonds (IT1 and IT2) (13) (Fig. 1A).

To monitor oxidative phases of HA folding, influenza virus infected cells were pulse-labeled for $4 \mathrm{~min}$ with 0.1 $\mathrm{mCi} / \mathrm{ml}\left[{ }^{35} \mathrm{~S}\right]-m e t h i o n i n e ~ a n d ~\left[{ }^{35} \mathrm{~S}\right]-$ cysteine and chased for $0,2,5,10$ or $20 \mathrm{~min}$ before alkylation with ice-cold PBS-NEM and lysis with 2\% CHAPS in HBS for preparation of the PNS. Cell lysates were supplemented with a polyclonal antibody that binds all oxidation forms of $\mathrm{HA}$ and also cross-reacts with the nuclear protein of influenza virus (NP, Fig. 1B). At 0 min chase HA equally populated the IT1 and IT2 intermediate forms and the NT form (Fig. 1B). With progression of the chase time, IT1 and IT2 were gradually converted in the NT form. The oxidative folding of HA was virtually terminated after a $10 \mathrm{~min}$ chase, when most labelled HA had acquired the native conformation. In the reducing gel, the information about the oxidative state of HA during the chase is lost. Intramolecular disulfides are in fact reduced by the DTT in the sample buffer so that HA migrates as a single polypeptide band (Fig. 1B, R).

The ER lectin calnexin ( $\mathrm{Cnx}$ ) associates transiently with monoglucosylated glycoproteins expressed in the lumen of the ER, thereby facilitating their maturation (4). To monitor transient association of HA with Cnx, the PNS were immunoprecipitated with a polyclonal antibody raised to the cytosolic tail of Cnx. Labelled Cnx has the same intensity in all lanes of the gel (Fig. 1C), the amount of labelled chaperone in the cell extracts was in fact the same throughout the chase. On the other hand, the amount of labelled cellular (arrowheads) and viral (HA) glycoproteins co-precipitated with $\mathrm{Cnx}$ decreased with progression of the chase. Association of Cnx with substrates is in fact transient, native polypeptides being eventually released from the chaperone to be transported along the secretory line to their final destination.
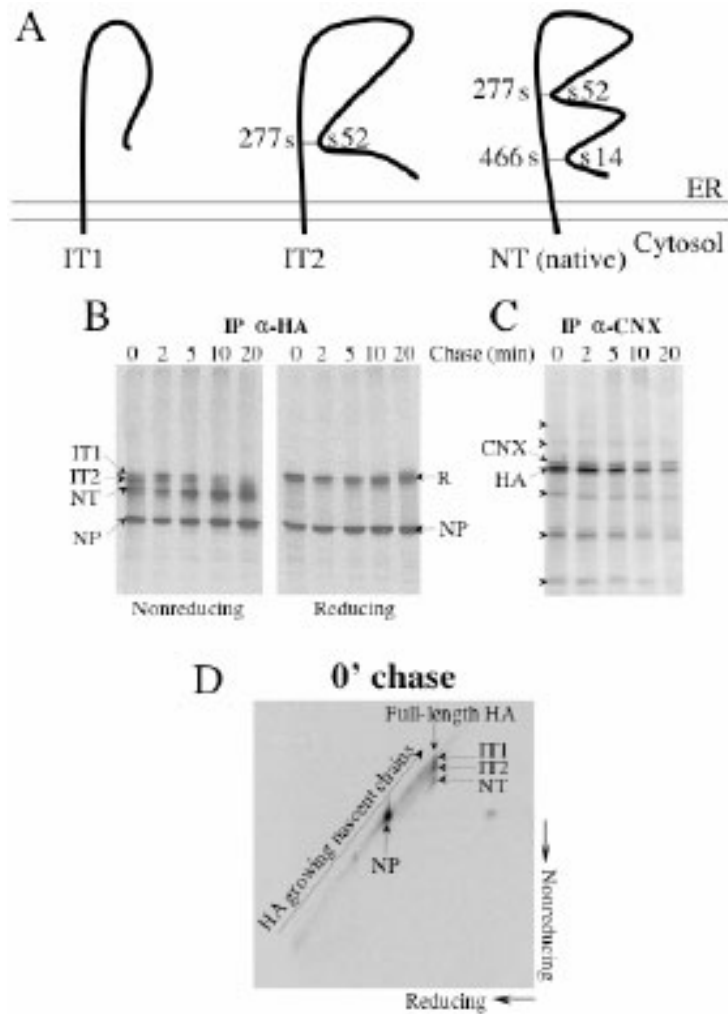

Fig. 1: Folding and association of influenza HA with calnexin. A. Schematics of major disulfide-bonds in influenza virus HA. Disulfides between cysteines 52-277 and cysteines 14-466 close two big loops. The NT form contains a full set of disulfides covalently linking the twelve HA cysteines, IT2 lacks the disulfide between cysteines 14 and 466, IT1 lacks both 52-277 and 14-466 disulfides. B. Oxidative HA folding analyzed in conventional SDS-PAGE under nonreducing and reducing conditions. $\mathbf{C}$. Transient association of cellular (arrowheads) and viral (HA) glycoproteins with Cnx. D. Analysis of co-translational HA oxidation in diagonal SDSPAGE.

Co-translational oxidation of HA was also analyzed in diagonal gels. Cells infected with influenza virus were pulse-labelled for $2.5 \mathrm{~min}$ with $\left.0.5 \mathrm{mCi} / \mathrm{ml}{ }^{[35} \mathrm{S}\right]-$ methionine and $\left[{ }^{35} \mathrm{~S}\right]$-cysteine. The pulse was interrupted by transferring dishes on ice and by a wash with the stop solution. PNS was immunoprecipitated with a polyclonal antibody to HA and analyzed by diagonal SDS-PAGE (Fig. 1D). Nascent HA chains were seen as a stripe of radioactivity from the lower-left corner (low molecular weight) to the upper-right corner (longer HA chains). Polypeptides without disulfide bonds ran on the gel 
diagonal, those with intramolecular disulfides (e.g. fulllength IT1, IT2 and NT) ran below the diagonal. The NP protein does not contain disulfides and, accordingly, it migrated on the gel diagonal. The presence of nascent chains below the diagonal clearly showed that formation of intramolecular disulfides started before completion of HA synthesis (Fig. 1D).

\section{ERAD of BACE457 $\triangle$ in mouse embryonic fibroblast}

Folding-defective polypeptides such as the variant of beta secretase expressed in the human pancreas (BACE457ム, (22)) are rapidly degraded to avoid accumulation of toxic protein aggregates in the ER lumen. To this end, they are translocated into the cytosol for proteasome-mediated proteolysis $(7,8)$. To determine kinetics of degradation of BACE, cells were transiently transfected with a BACE expression plasmid, pulselabelled for $10 \mathrm{~min}$ and chased for different times. The PNS were immunoprecipitated with a specific antibody to BACE. The nonreducing gel showed that cellular BACE was in different oxidation states. High molecular weight disulfide-bonded aggregates (DBA) containing misfolded BACE hardly entered the nonreducing running gel and were visible at the top of the gel (Fig. 2A). Monomeric BACE entered in the gel and was clearly separated in three distinct oxidation forms (arrows). These progressively disappeared during the chase to be in part converted in DBA and in part degraded from the ER. Sample reduction disassembled DBA and converted all oxidation forms of BACE in a single polypeptide band (BACE in the reducing gel (Fig. 2A)).

The heterogeneity of BACE conformations makes analysis of degradation kinetics in nonreducing gels difficult. ERAD kinetics can, on the other hand, precisely be quantified in the reducing gel, where BACE migrated as a single (Fig. 2A).

Small variations of the experimental procedures described above, for example the expression of the model proteins in cells exposed to inhibitory compounds or depleted of selected molecular chaperones, allows establishing the mechanisms of ERAD with identification of luminal and cytosolic factors involved. In the example shown in Fig. 2B, cell-permeable inhibitors of protein degradation from the ER were added in the starvation, pulse and chase media to specifically inactivate the cytosolic proteasome (MG132 (M)) or the ER alphamannosidase I (kifunensine $(\mathrm{K})$ ). Both inhibitors substantially delayed degradation of BACE as shown by the higher amount of labelled BACE remaining at the end of the $90 \mathrm{~min}$ chase, thus showing the involvement of the proteasome and the importance of mannose trimming for ERAD.

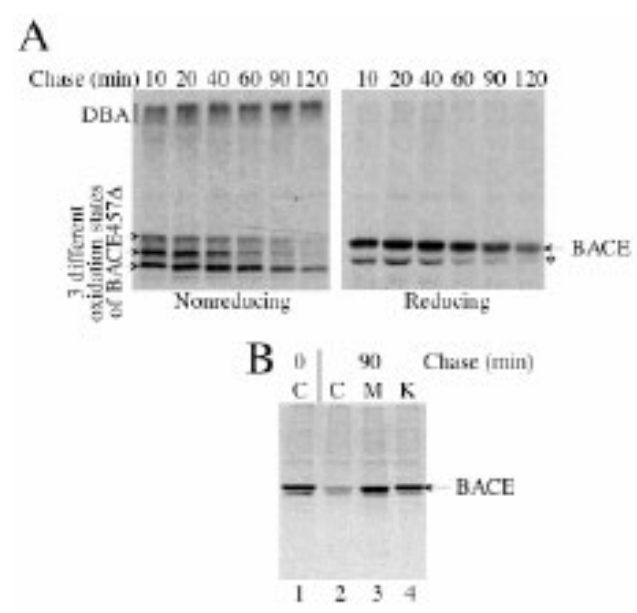

Fig. 2: Analysis of ER-associated degradation of BACE457D. A. Labeled BACE was in different oxidation states; monomeric BACE entered in the nonreducing running gel as 3 distinct oxidation forms (arrows), oligomeric BACE remained at the top of the nonreducing gel in disulfide-bonded aggregates (DBA). In the reducing gel DBA and the several BACE oxidation forms were reduced in a single BACE polypeptide (BACE). The asterisk shows non-glycosylated BACE. B. After 90 min chase BACE was efficiently degraded (compare the intensity of labeled BACE at 0 (lane 1) and at 90 min chase (lane 2). MG132 (M) and kifunensine (K) substantially inhibited BACE degradation (lanes 3 and 4 , respectively).

In summary, we described here experiments in living cells designed to investigate molecular mechanisms regulating the maturation of folding-competent and the degradation of folding-defective polypeptides. These investigations rely on enhanced expression of model polypeptides obtained by appropriately transfecting or infecting mammalian cultured cells. The fate of the radioactively labelled, newly synthesized, model proteins is monitored by conventional or diagonal gel electrophoresis. The approaches highlighted in this paper can easily be adapted and implemented to compare protein fates in cells with a complete set of chaperones versus cells deleted of a selected chaperone or exposed to substances that inhibit chaperone or enzyme activities.

Soldà et al. - Analyzing folding and degradation of metabolically labelled polypeptides by conventional and diagonal sodium dodecyl sulfate polyacrylamide gel electrophoresis

www.biologicalprocedures.com 


\section{ACKNOWLEDGMENTS}

M.M. is supported by grants from Max Cloetta Foundation, Foundation for Research on Neurodegenerative Diseases, Swiss National Center of Competence in Research on Neural Plasticity and Repair, Swiss National Science Foundation, Telethon and Synapsis/Bangerter-Rhyner Foundation.

\section{REFERENCES}

1. Gething MJ, Sambrook J. Protein folding in the cell. Nature 1992; 355:33-45.

2. Parodi AJ. Role of $\mathrm{N}$-oligosaccharide endoplasmic reticulum processing reactions in glycoprotein folding and degradation. Biochem J 2000; 348:1-13.

3. Ellgaard L, Ruddock LW. The human protein disulphide isomerase family: substrate interactions and functional properties. EMBO Rep 2005; 6:28-32.

4. Ellgaard L, Molinari M, Helenius A. Setting the standards: quality control in the secretory pathway. Science 1999; 286:1882-1888.

5. Hebert DN, Garman SC, Molinari M. The glycan code of the endoplasmic reticulum: asparaginelinked carbohydrates as protein maturation and quality-control tags. Trends Cell Biol 2005; 15:364-370.

6. Schroder M, Kaufman RJ. The Mammalian unfolded protein response. Annu Rev Biochem 2005; 74:739-789.

7. Meusser B, Hirsch C, Jarosch E, Sommer T. ERAD: the long road to destruction. Nat Cell Biol 2005; 7:766772.

8. McCracken AA, Brodsky JL. Evolving questions and paradigm shifts in endoplasmic-reticulum-associated degradation (ERAD). Bioessays 2003; 25:868-877.

9. Ou WJ, Cameron PH, Thomas DY, Bergeron JJ. Association of folding intermediates of glycoproteins with calnexin during protein maturation. Nature 1993; 364:771-776.

10. Hammond C, Helenius A. Folding of VSV G protein: sequential interaction with $\mathrm{BiP}$ and calnexin. Science 1994; 266:456-458.

11. Nauseef WM, McCormick SJ, Clark RA. Calreticulin functions as a molecular chaperone in the biosynthesis of myeloperoxidase. J Biol Chem 1995; 270:4741-4747.

12. Molinari M, Helenius A. Glycoproteins form mixed disulphides with oxidoreductases during folding in living cells. Nature 1999; 402:90-93.
13. Braakman I, Hoover-Litty H, Wagner KR, Helenius A. Folding of influenza hemagglutinin in the endoplasmic reticulum. J Cell Biol 1991; 114:401-411.

14. Chen W, Helenius J, Braakman I, Helenius A. Cotranslational folding and calnexin binding during glycoprotein synthesis. Proc Natl Acad Sci USA 1995; 92:6229-6233.

15. Cabibbo A, Pagani M, Fabbri M, Rocchi M, Farmery MR, Bulleid NJ, Sitia R. ERO1-L, a human protein that favors disulfide bond formation in the endoplasmic reticulum. J Biol Chem 2000; 275:48274833.

16. Liu Y, Choudhury P, Cabral CM, Sifers RN. Oligosaccharide modification in the early secretory pathway directs the selection of a misfolded glycoprotein for degradation by the proteasome. $J$ Biol Chem 1999; 274:5861-5867.

17. Molinari M, Eriksson KK, Calanca V, Galli C, Cresswell P, Michalak M, Helenius A. Contrasting Functions of Calreticulin and Calnexin in Glycoprotein Folding and ER Quality Control. Mol Cell 2004; 13:125-135.

18. Gallione CJ, Rose JK. A single amino acid substitution in a hydrophobic domain causes temperature-sensitive cell-surface transport of a mutant viral glycoprotein. J Virol 1985; 54:374-382.

19. Helenius A, Simons K. Solubilization of membranes by detergents. Biochim Biophys Acta 1975; 415:29-79.

20. Sommer A, Traut RR. Diagonal polyacrylamidedodecyl sulfate gel electrophoresis for the identification of ribosomal proteins crosslinked with methyl-4- mercaptobutyrimidate. Proc Natl Acad Sci USA 1974; 71:3946-3950.

21. Molinari M, Helenius A. Analyzing cotranslational protein folding and disulfide formation by diagonal sodium dodecyl sulfate-polyacrylamide gel electrophoresis. Methods Enzymol 2002; 348:35-42.

22. Molinari M, Calanca V, Galli C, Lucca P, Paganetti P. Role of EDEM in the release of misfolded glycoproteins from the calnexin cycle. Science 2003; 299:1397-1400.

Soldà et al. - Analyzing folding and degradation of metabolically labelled polypeptides by conventional and diagonal sodium dodecyl sulfate polyacrylamide gel electrophoresis 


\section{PROTOCOLS}

\section{1) Amplification of the signal}

Mammalian tissue culture cells are seeded in 6-cm-diameter dishes (one dish for each chase time-point to be analyzed) the day before the experiment. Cells are grown in the appropriate cell culture medium in a $37^{\circ} \mathrm{C}$ humidified $5 \% \mathrm{CO}_{2}$ incubator.

Transfection: Several transfection protocols are available depending on the transfection reagent and the cell line used for the experiments. Here is the protocol used in our lab for adherent mammalian cells. The day of the transfection cells have to be at $90-100 \%$ confluency. Cell culture media is replaced with $3 \mathrm{ml}$ transfection media. The plasmid-ofinterest $(4 \mu \mathrm{g})$ is diluted with $300 \mu \mathrm{l}$ Optimem in a $5 \mathrm{ml}$ plastic tube. In a second tube, $15 \mu \mathrm{l}$ of Lipofectamine ${ }^{\mathrm{TM}} 2000$ are re-hydrated for $5 \mathrm{~min}$ in $300 \mu \mathrm{l}$ Optimem. The solutions containing DNA and Lipofectamine ${ }^{\mathrm{TM}} 2000$ are mixed and left $20 \mathrm{~min}$ at room temperature before addition to the cells. Pulse-chase experiment is usually performed the next day.

Viral infection: Influenza virus (the model glycoprotein is then influenza hemagglutinin, HA), vesicular stomatitis virus ( $\mathrm{G}$ protein and its temperature-sensitive mutant ts045 (18)) and Semliki forest virus (E1 and p62) have been widely used to characterize the secretory pathway. Each dish is first washed with PBS and then incubated at room temperature for $45 \mathrm{~min}-1 \mathrm{hr}$ with $0.8 \mathrm{ml}$ of inoculum. The inoculum is replaced with $2.5 \mathrm{ml} /$ dish of cell culture medium and dishes are left for 4 additional hours at $37^{\circ} \mathrm{C}$ in a $5 \% \mathrm{CO}_{2}$ incubator.

\section{2) Pulse-Chase (all steps at $37^{\circ} \mathrm{C}$ )}

Cells are washed with PBS (2 ml/dish) and starved in $1.5 \mathrm{ml} /$ dish of starvation medium $\left(15-30 \mathrm{~min}\right.$ in a $5 \% \mathrm{CO}_{2}$ incubator). Dishes are then moved onto a non-floating rack placed in a water-bath. For metabolic labelling the starvation medium is replaced with $1 \mathrm{ml} /$ dish of pulse medium. Metabolic labelling is stopped by addition of $1 \mathrm{ml}$ chase medium quickly aspirated (aspirate in suitable flask to safely collect the radioactive medium) and chase is started by the addition of $2 \mathrm{ml} /$ dish of chase medium.

\section{3) Sample alkylation and cell lysis (all steps at $\left.4^{\circ} \mathrm{C}\right)$}

After the selected chase time, each dish is transferred on ice and the chase medium is replaced with ice-cold PBS/20 mM NEM (Stop solution). NEM is a cell-permeable alkylating agent that rapidly permeates in cells and blocks reactivity of free SH-groups of cysteines. Cells are incubated for 1-5 $\mathrm{min}$ in the stop solution. The solution is then aspirated as dry as possible before addition of $0.8 \mathrm{ml} /$ dish of cell lysis buffer. When non-covalent interactions such as those often occurring between protein-of-interest and ER-resident molecular chaperones are investigated, an isotonic, buffered solution containing a protease inhibitors cocktail (CLAP and PMSF) and 2\% CHAPS as detergent is preferred. CHAPS is a zwitterionic detergent that solubilizes plasma and endomembranes, whilst the nuclear membrane remains intact. After 20-30 min on ice, the cell lysate is collected in an Eppendorf tube and centrifuged at $10600 \mathrm{~g}$ for $10 \mathrm{~min}$ to pellet the nuclei. The post nuclear supernatant (PNS) is aliquoted, snap-frozen in liquid nitrogen and stored at $-80^{\circ} \mathrm{C}$.

Note: a) Several classes of detergents are used to solubilize biological membranes. 1. Ionic: used for the total disruption of a membrane and the dissociation of every component protein into monomers (e.g. Sodium dodecyl sulfate (SDS), an anionic detergent that denatures and inactivates most membrane proteins). 2. Non-ionic: used to solubilize membrane proteins in a much gentler fashion, often preserving native subunit structure and enzymatic

Soldà et al. - Analyzing folding and degradation of metabolically labelled polypeptides by conventional and diagonal sodium dodecyl sulfate polyacrylamide gel electrophoresis

www.biologicalprocedures.com 
activity (e.g. TRITON X-100 ${ }^{\circledR}$, Tween 20). 3. Zwitterionic (e.g. CHAPS). For a comprehensive review on solubilization of membranes by detergent please refer to (19). b) Calcium chelating agents such as EDTA should be avoided when association between the ER resident lectins calnexin and calreticulin with their substrates are investigated since these associations are calcium-dependent c) Association between the chaperone BiP/GRP78 and substrates is preserved by cell lysis only in the presence of 10 units/dish of Apyrase (Sigma) that rapidly depletes cellular ATP.

\section{4) Immunoprecipitation (all steps at $4^{\circ} \mathrm{C}$ )}

A fraction, usually 1:5-1:10 of the total PNS from each dish, is mixed with $100 \mu \mathrm{PAB}$ and an antibody specific for the protein-of-interest or for a selected chaperone to determine if this is associated with the protein-of-interest. Samples are incubated with slow rotation for 1-4 hr. PAB are then pelleted at $1800 \mathrm{~g}$ for $2 \mathrm{~min}$ in an Eppendorf centrifuge; the supernatant is aspirated with a flat-tip (unbound material to be discharged) and beads are resuspended in $1 \mathrm{ml}$ of washing buffer. Samples are shaken for at least $5 \mathrm{~min}$ in Eppendorf tube shaker. Washing is repeated for 3 times. After the last wash, the washing solution is carefully aspirated and, for conventional SDS-PAGE, $30 \mu \mathrm{l}$ of $3 \mathrm{X}$ sample buffer are added to the dry PAB. Samples are heated at $95^{\circ} \mathrm{C}$ for $5 \mathrm{~min}$. Half of the sample is transferred in another Eppendorf tube containing $100 \mu \mathrm{M}$ fin of DTT. For diagonal SDS-PAGE the samples are boiled in nonreducing sample buffer to be processed for first dimension capillary electrophoresis (see below).

\section{5) Conventional SDS-PAGE}

One $\mathrm{mm}$ thick polyacrylamide separating and stacking minigels are prepared, adjusting the acrylamide percentage to the apparent molecular weight of the protein-of-interest (e.g. for influenza virus HA, a glycoprotein of about $80 \mathrm{kDa}$ we use $10 \%$ polyacrylamide gels, for Semliki Forest virus E1 $(40 \mathrm{kDa})$ and p62 $(60 \mathrm{kDa})$ we use $12 \%$ polyacrylamide gels). Allow at least $90 \mathrm{~min}$ of polymerization time before running the gels. When loading the gels note that at least one empty lane should separate nonreducing and reducing samples to avoid reduction of the former during the run. The empty lanes are filled with sample buffer. Gels are run at $200 \mathrm{~V}$ until the dye front exits (about $45 \mathrm{~min}$ ). The gel is then stained with Coomassie blue for $5 \mathrm{~min}$ and destained until molecular weight markers and endogenous proteins (or antibody chains in case immunoprecipitates are analyzed) are clearly visible. Stain and destain solutions can be reused several times. The gel is dried on a $0.5 \mathrm{~mm}$ Whatman filter paper in a vacuum/heated gel dryer and exposed to film or to a Phosphor imaging screen. Overnight exposures are often sufficient to clearly distinguish labelled polypeptides.

\section{6) Diagonal SDS-PAGE}

Diagonal gels are 2D gels originally used to analyze chemically cross-linked ribosomal components (20) and subsequently adapted to investigate cotranslational events and oxidative phases of glycoprotein folding (reviewed in (21)). The first dimension is run under nonreducing conditions in a glass capillary tube of $7 \mathrm{~cm}$ length and an internal diameter of $1.3 \mathrm{~mm}$. For the second dimension, the gel is extruded from the glass tube, boiled in reducing sample buffer and placed onto a conventional slab gel.

First dimension: capillary glass tubes are washed with ethanol and, then distilled water and then dried in an oven for at least $1 \mathrm{hr}$ at $70^{\circ} \mathrm{C}$ or at room temperature for at least $24 \mathrm{hr}$. Ten tubes are put in a plastic cylinder few millimeters shorter than the capillary tubes themselves, to facilitate manipulations. The tube can easily be prepared by cutting with a knife the bottom of a $15 \mathrm{ml}$ Falcon. The Falcon's lid is then the base of the cylinder. The acrylamide solution prepared as for a normal nonreducing SDS-PAGE is poured rapidly after addition of ammonium persulfate and TEMED in the plastic cylinder (it enters the glass tubes per capillarity, from the bottom). A few $\mu$ l of isobutanol or distilled water is carefully added to the above gels in each capillary tube and polymerization is allowed for at least 90

Soldà et al. - Analyzing folding and degradation of metabolically labelled polypeptides by conventional and diagonal sodium dodecyl sulfate polyacrylamide gel electrophoresis

www.biologicalprocedures.com 
min. The isobutanol or water is replaced with $30 \mu \mathrm{l}$ of stacking gel/capillary tube. The capillary tubes can be stored for several weeks at $4^{\circ} \mathrm{C}$ in a humid chamber.

The first dimension is run in a BioRad 2D gel system according to the manufacturer's instructions. The glass capillary tubes are attached to the sample-reservoir with flexible plastic tube connectors. The lower tank is filled with approximately $800 \mathrm{ml}$ of SDS-PAGE running buffer. Air bubbles are carefully removed from the tubes and samples are loaded with a Hamilton syringe (for better resolution, not more than $15 \mu \mathrm{l}$ of sample are loaded). The first dimension is run at $100 \mathrm{~V}$, in a cold room to avoid excessive heating of the capillary tubes, until the blue front runs out. At the end of the run, the gel is carefully extruded from the glass capillary tube using a tube gel ejector (BioRad) connected to a syringe filled with running buffer. The capillary gel is boiled for $10 \mathrm{~min}$ in an Eppendorf tube containing $800 \mu \mathrm{l}$ of reducing sample buffer.

For the second dimension the capillary gel is carefully slid between the glass plates of a conventional slab gel with the help of a clean spatula. The second dimension is run at $200 \mathrm{~V}$ constant voltage until the dye front reaches the bottom of the gel. The gel is stained with Coomassie blue for $5 \mathrm{~min}$, destained and dried on a $0.5 \mathrm{~mm}$ Whatman filter paper. The gel is then exposed on Biomax film. Very little radioactivity is incorporated in growing nascent chains, thus long exposures are often required. 